\title{
Geography Lessons: Scrutinizing State-by-State Differences in Inpatient Gastroparesis Care
}

\author{
William L. Hasler
}

Published online: 11 August 2013

(C) Springer Science+Business Media New York 2013

Gastroparesis is defined as a slowing of gastric emptying, a condition that has been associated with nausea, vomiting, and related symptoms. Few papers have addressed its prevalence and incidence and have characterized factors related to health care utilization for this condition. A recent population-based analysis reported prevalence per 100,000 for definite gastroparesis (defined by typical symptoms with documented delayed gastric emptying) of 9.6 for men versus 37.8 for women [1]. Age-adjusted incidences per 100,000 person-years calculated from the same population were 2.4 for men and 9.8 for women. Nevertheless, these data may not be generalizable due to its unique locale, Olmsted County, Minnesota, a highly educated, relatively homogeneous population medically well served by the Mayo Clinic. Furthermore, the impact of geography, demographics, and socioeconomic factors on hospitalization rates for gastroparesis and on other parameters of health resource usage has not heretofore been reported in the medical literature.

The article by Bielefeldt in this issue represents an important addition to the literature, providing voluminous new information relating to regional profiles of inpatient care for gastroparesis (Table 1). The study utilized the State Inpatient Databases, which accumulate demographic and clinical information from $90 \%$ of hospitalizations in 46 states. For this investigation, the International Diagnosis Code (ICD-9) for gastroparesis (536.3) was queried from 2007 through 2010 for 35 states. The most striking findings were the greater-than-fourfold differences in admission

\section{W. L. Hasler $(\bowtie)$}

Division of Gastroenterology, University of Michigan Health System, 3912 Taubman Center, 5362, Ann Arbor, MI 48109, USA

e-mail: whasler@med.umich.edu; whasler@umich.edu rates for this condition. It is possible that states with high admission percentages are the main contributors to the previously reported increase in hospitalizations for gastroparesis [2]. Twofold geographic differences in length of stay and nursing home transfers were also observed. Among demographic factors, higher hospitalization rates were related to female sex, greater state population, lower incomes, admission to large centers, and Medicare coverage. Clinically, heart failure was associated with increased admissions, whereas diabetes was inversely related to hospitalizations. Admission data correlated roughly with rates of endoscopy and in older individuals, gastrostomy placement, both of which also showed wide state-to-state variability. Conversely, enteral or parenteral nutrition initiation did not strictly relate to hospitalization rates. Finally, higher numbers of overall admissions but lower percentages of admissions with gastroparesis as a primary diagnosis were associated with inpatient mortality as were age $>65$ years, female sex, poverty, and endoscopies. The author concluded that disease-dependent and socioeconomic factors contribute to regional differences in inpatient health care for gastroparesis.

Although the analyses by Bielefeldt were broad-ranging, other administrative factors related to differences in coding may have contributed to apparent regional variations in inpatient care of gastroparesis. The author did attempt to address this issue by comparing ICD-9 coding for gastroparesis versus dyspepsia. Yet, annual admissions for dyspepsia were only $2-3 \%$ of those for gastroparesis in this study. Although hospitalization data for functional dyspepsia are lacking for the United States, its prevalence in the community exceeds $5 \%$ and its inpatient costs are greater than threefold higher than for non-dyspeptics [3]. Thus, it is likely the coding of this disorder within this database did not consistently capture all functional 
Table 1 Contributing factors to regional disparities in inpatient care for gastroparesis

\begin{tabular}{|c|c|c|}
\hline Observations & Factors defined to relate to geographic variation & $\begin{array}{l}\text { Other observations and contributors to } \\
\text { geographic variation }\end{array}$ \\
\hline $\begin{array}{l}\text { Greater-than-fourfold state-to-state range of } \\
\text { gastroparesis admissions } \\
\text { Nearly fourfold range of endoscopy, gastrostomy, } \\
\text { and supplemental nutrition initiation } \\
\text { Nearly fourfold range of inpatient mortality, } \\
\text { twofold range of length of stay, and >twofold } \\
\text { range of nursing home transfer }\end{array}$ & $\begin{array}{l}\text { Higher admissions correlated with: female sex, } \\
\text { higher state population, lower income } \\
\text { Admission to for-profit or large hospitals, } \\
\text { Medicare coverage } \\
\text { Heart failure and inversely related to diabetes } \\
\text { Numerous socioeconomic factors also correlated } \\
\text { with other parameters of resource usage and } \\
\text { hospitalization outcomes }\end{array}$ & $\begin{array}{l}\text { Probable variable capture of idiopathic } \\
\text { cases due to differential diagnosis of } \\
\text { functional dyspepsia } \\
\text { Probable other cases not captured due to } \\
\text { other ICD-9 codes not considered } \\
\text { Probable variable regional utilization } \\
\text { rates of gastric scintigraphy } \\
\text { Probable regional variabilities on how a } \\
\text { diagnosis of gastroparesis is made }\end{array}$ \\
\hline
\end{tabular}

dyspeptics across states. Furthermore, the authors did not query the more than ten other relevant ICD-9 codes. Consequently, depending on the diagnostic evaluation or the attentiveness of the inpatient provider, it is probable that a significant number of patients were not captured by these databases.

Most older studies report comparable prevalence of diabetic and idiopathic gastroparesis, with each subgroup comprising one-quarter to one-third of the total cohort [4]. Still, nearly two-thirds of patients with gastroparesis recruited into a recent, large multicenter registry of gastroparesis had idiopathic disease [5], possibly as a consequence of two opposing trends: Firstly, diabetic care now focuses more intensively on improving glycemic control, potentially reducing progression to severe diabetic gastroparesis. Secondly, increased use of scintigraphy in patients with gastroparesis-like symptoms may have increased the diagnosis of idiopathic disease. Bielefeldt suggest that the regional disparities in hospitalizations for gastroparesis may be driven by the subset with idiopathic gastroparesis, since the hospitalization percentages for diabetic gastroparesis were geographically more uniform. This could offer a partial explanation for the author's observed inverse relationship of hospitalizations with diabetes.

The classification of patients with dyspeptic symptoms and delayed gastric emptying as having idiopathic gastroparesis or one of the Rome Foundation diagnoses such as functional dyspepsia, chronic idiopathic nausea, functional bloating, or functional vomiting is controversial. Ninetyone percent of a large cohort of well-characterized idiopathic gastroparesis patients also satisfied Rome III criteria for the postprandial distress variant of functional dyspepsia [5]. Janssen and colleagues highlighted this controversy commenting "the existence of a separate nomenclature for idiopathic gastroparesis has more to do with the fact that delayed emptying was historically the first hypothesis to be addressed in unexplained upper GI symptoms, and with the widespread availability of gastric emptying testing, than with its relevance to clinical management" [6]. This would suggest that apparent differences in admissions for idiopathic gastroparesis may have stemmed from increased utilization of scintigraphy or preferential application of gastroparesis diagnoses for non-diabetics with appropriate symptoms. Although Bielefeldt quantified endoscopy, geographic variation in the use of gastric scintigraphy was not reported. Though admission percentages did not correlate with the number of gastroenterologists, the number of physicians with focused expertise in gastrointestinal motility disorders was unavailable, due to limitations of the State Inpatient Databases.

Finally, this comprehensive report calls into question the regional variation on the diagnosis of gastroparesis. Standardized gastric scintigraphy protocols have been adopted by only by the minority of facilities in most states [7]. Additionally, alternate methods of scintigraphic interpretation may promote discordant gastroparesis diagnoses. For example, delayed half times of emptying and 4-h gastric retention rates may be discordant in one-third of cases. Furthermore, clinicians commonly diagnose gastroparesis in the absence of objective emptying measures. In diabetics, symptom severity correlates poorly with prolonged gastric retention [8]. Even in research institutions, gastroparesis is often diagnoses solely on the basis of typical symptoms [1].

Nevertheless, the careful analyses performed by Bielefeldt emphasize important geographic variations in the inpatient care of gastroparesis. Similar state-by-state variations in outpatient resource utilization likely exist. One purpose of peer-reviewed literature and the major medical societies is to promote a uniform high level of care for any given gastrointestinal disease. These observations provide a foundation for the next series of analyses — characterizing regional differences in management protocols, which will determine if variations exist in drug prescriptions and nonmedication-based therapies and associated treatment benefits. 


\section{References}

1. Jung HK, Choung RS, Locke GR, et al. The incidence, prevalence, and outcomes of patients with gastroparesis in Olmsted County, Minnesota, from 1996 to 2006. Gastroenterology. 2009;136: $1225-1233$.

2. Wang YR, Fisher RS, Parkman HP. Gastroparesis-related hospitalizations in the United States: trends, characteristics, and outcomes, 1995-2004. Am J Gastroenterol. 2008;103:313-322.

3. Brook RA, Kleinman NL, Choung RS, et al. Functional dyspepsia impacts absenteeism and direct and indirect costs. Clin Gastroenterol Hepatol. 2010;8:498-503.

4. Soykan I, Sivri B, Sarosiek I, et al. Demography, clinical characteristics, psychological and abuse profiles, treatment, and long-term follow-up of patients with gastroparesis. Dig Dis Sci. 1998;43:2398-2404.
5. Parkman HP, Yates K, Hasler WL, et al. Clinical features of idiopathic gastroparesis vary with sex, body mass, symptom onset, delay in gastric emptying, and gastroparesis severity. Gastroenterology. 2011;140:101-115.

6. Janssen P, van Oudenhove L, Bisschops R, Tack J. Idiopathic gastroparesis or functional dyspepsia with delayed gastric emptying: where is the difference? Gastroenterology. 2011;140: 2145-2146.

7. Abell TL, Camilleri M, Donohoe K, et al. Consensus recommendations for gastric emptying scintigraphy: a joint report of the American Neurogastroenterology and Motility Society and the Society of Nuclear Medicine. Am J Gastroenterol. 2008;103: 753-763.

8. Pasricha PJ, Colvin R, Yates K, et al. Characteristics of patients with chronic unexplained nausea and vomiting and normal gastric emptying. Clin Gastroenterol Hepatol. 2011;9:567-576. 